\title{
Wo geht's hin in der Dialysewelt?
}

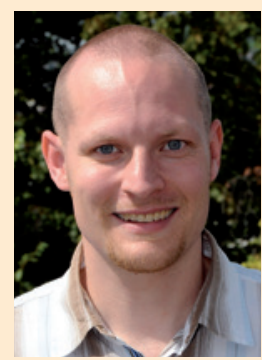

Christian Schäfer, Stuttgart
Ja, wohin geht's denn nun? Wird die Dialyse-Sachkosten-Pauschale nach der ersten Absenkung am 1. Juli 2013 am 1. Januar 2015 ein zweites Mal abgesenkt (dieses Mal um 80 Millionen Euro jährlich), geht es wohl weiter in die falsche Richtung: Die dem Einheitlichen Bewertungsmaßstab (EBM) zugrunde gelegte Struktur der Praxiskosten unterschätzt die tatsächliche Struktur um etwa ein Viertel. Das sagte Dr. sc. pol. Thomas Drabinski, Leiter des Instituts für Mikrodaten-Analyse (IfMDA) in Kiel, gegenüber dem Deutschen Ärzteblatt, nachdem die Ergebnisse des vom Verband Deutscher Nierenzentren (DN) in Auftrag gegebenen Gutachtens vorlagen. In Geldbeträgen ausgedrückt besagen die Analysen laut IfMDA, dass die jährlichen „technischen Kosten“ einer Dialysepraxis bzgl. gesetzlich versicherter Patienten bei ca. 991000 Euro pro Versorgungsauftrag liegen - und nicht bei etwa 740000 Euro, wie im EBM angenommen. Berücksichtigt wurden bei der Befragung ca. 800 Ärzte aus mehr als 330 Praxen, die DN-Mitglied sind sowie Dialyse-Sachkosten-Pauschalen und ärztliche Pauschalen über Kassenärztliche Vereinigungen abrechnen. Ungefähr 85\% der Befragten gaben die erforderlichen Auskünfte.

Die jährliche Differenz von ungefähr 251000 Euro ist erheblich. Bedenkt man die Auswirkungen der Pauschalenkürzung, wiegt sie allerdings noch schwerer als auf den ersten Blick gedacht: Um die fehlenden Einnahmen auszugleichen, liegen für die Praxisinhaber Einsparungen beim Personal nahe. Leider bedeutet dies wohl, dass teurere, aber eben auch kompetentere nephrologische Fachpflegekräfte nach und nach durch günstigere Mitarbeiter mit einem kleineren Wissen rund um die aufwendigen Nierenersatzbehandlungen und den Patientenumgang ersetzt werden. Was dies für die Patientensicherheit und die Dialysequalität bedeutet, ist nicht schwer zu prognostizieren.

Die Arbeitsgemeinschaft für neophrologisches Personal e.V. (AfnP) hat lobenswerterweise auf die sich verändernden Rahmenbedingungen reagiert: Sie bietet in diesem Jahr die „Dialyse Basic Days“ statt des AfnP-Symposiums an. Das Ziel der Veranstaltung am 26./27. September in Fulda ist es, in Vorträgen und Workshops praxisorientiert Grundlagen zur Dialyse zu vermitteln. Details hierzu können Sie auf Seite 286 in dieser Ausgabe der Dialyse aktuell nachlesen. Die „Dialyse Basic Days“ können dabei helfen, mangelnde Kenntnisse und Erfahrungen bei neu dazu gestoßenem Dialysepersonal etwas auszugleichen. Aber dies ist natürlich auch nur eine Symptombekämpfung, die nichts an den Ursachen für den Umbau der Dialyseteams ändern kann.

Zurück zur drohenden zweiten Absenkung der Dialyse-Sachkosten-Pauschale: Ob sie tatsächlich Realität wird, hängt v.a. von den Verhandlungen der Kassenärztlichen Bundesvereinigung (KBV) mit dem Spitzenverband der gesetzlichen Krankenversicherung (GKV) ab. Immerhin äußerte sich Dr. rer. pol. Ulrich Casser, KBV-Honorardezernent, laut dem Deutschen Ärzteblatt hierzu dahingehend, dass die KBV die IfMDA-Daten in die Verhandlungen mit der GKV einfließen lässt und einer weiteren Absenkung nicht zustimmen werde. Wie auch schon im Editorial in Dialyse aktuell 6/2013 erwähnt, war die Datenlage, auf deren Basis KBV und GKV die Absenkungsschritte beschlossen hatten, sehr dürftig. Die IfMDA-Ergebnisse sind, wie auch Casser erwähnte, dagegen wohl deutlich näher an der Realität der Dialyseversorgung.

Sie sehen, es gibt also noch Grund zur Hoffnung! Ich wünsche Ihnen in diesem Sinne eine angenehme Lektüre dieser Ausgabe der Dialyse aktuell und schöne, aber nicht zu heiße Sommertage! 